\title{
Hopping-resolved electron-phonon coupling in bilayer graphene
}

\author{
E. Cappelluti ${ }^{1,2}$ and G. Profeta ${ }^{3,4}$ \\ ${ }^{1}$ Instituto de Ciencia de Materiales de Madrid, ICMM-CSIC, Cantoblanco, E-28049 Madrid, Spain \\ ${ }^{2}$ Institute for Complex Systems (ISC), CNR, U.O.S. Sapienza, v. dei Taurini 19, 00185 Roma, Italy \\ 3 Dipartimento di Fisica, Università dell'Aquila, Via Vetoio 10, I-67100 L'Aquila, Italy \\ 4 CNR-SPIN Via Vetoio 10, I-67100 L'Aquila, Italy
}

(Dated: November 8, 2018)

\begin{abstract}
In this paper we investigate the electron-phonon coupling in bilayer graphene, as a paradigmatic case for multilayer graphenes where interlayer hoppings are relevant. Using a frozen-phonon approach within the context of Density Functional Theory (DFT) and using different optical phonon displacements we are able to evaluate quantitatively the electron-phonon coupling $\alpha_{i}$ associated with each hopping term $\gamma_{i}$. This analysis also reveals a simple scaling law between the hopping terms $\gamma_{i}$ and the electron-phonon coupling $\alpha_{i}$ which goes beyond the specific DFT technique employed.
\end{abstract}

\section{INTRODUCTION}

Since its discovery, a formidable amount of work has been devoted to investigate the electronic and structural properties of single-layer and multi-layer graphenes. The electron-phonon interaction has in particular attracted a huge interest for its role in controlling the charge transport, $\underline{\underline{1}-\underline{5}}$ for providing a powerful interface between electronic and structural properties,,$\underline{\underline{6}-\underline{9}}$ and also because phonon resonances in Raman and infrared spectroscopies, triggered by the electron-phonon interaction, represent a useful way to characterize graphenic samples and to reveal interesting unconventional effects. ${ }^{10}-17$

On the theoretical level, tight-binding (TB) models are of fundamental importance since they have been shown to catch almost all the electronic features in this systems. The vast majority of works based on TB in literature $\frac{18}{18}$ employ a simple two-parameter TB model, where only the nearest neighbor in-plane hopping $\gamma_{0}$ and the nearest neighbor vertical hopping $\gamma_{1}$ are considered, although, when needed, higher order TB terms are included to reproduce more detailed features $\underline{\underline{19}} \underline{27}$ Most important in bilayer graphene, borrowing the terminology from bulk graphite,, 28 on the atoms $\mathrm{B} 1$ and $\mathrm{A} 2$, and the hopping terms $\gamma_{3}$, operative between the atoms A1-B2, and $\gamma_{4}$ between atom couples A1-A2 and B1-B2 (see Fig. 1). Such terms represent thus the basilar ingredients to build a TB model in multilayer graphene with both Bernal (ABAB...) and rhombohedral (ABCA...) stacking. For instance, the hopping term $\gamma_{3}$ was shown to be related to the trigonal warping and, $\stackrel{28}{=}$ in bilayer systems, to the generation of new Dirac points at finite momentum close to the $\mathrm{K}$ point.

Tight-binding models are also widely employed to investigate the electron-phonon interaction. Focusing on the single-layer graphene, the most relevant in-plane lattice vibrations are related to the modulation of the nearest neighbor hopping $\gamma_{0} \stackrel{18}{=}$ Within this context, for instance, the optical properties on the $E_{2 g}$ phonon band at $\omega \approx 0.2 \mathrm{eV}$ have been throughout investigated $29-33$ as well as the effects on the electronic structure of the long wavelength acoustic modes associated with the ripples,$\frac{5,6,8}{1}$ Modeling of the electron-phonon interaction in multilayer graphene is also commonly discussed on the basis of the modulation of the $\gamma_{0}$ hopping. Among other things, this kind of analysis was useful to show the robustness of the Dirac points ${ }^{34}$ upon lattice distortions, in single- as well in multi-layer systems $\frac{35,36}{, 36}$

Alternative to tight-binding model, Density Functional Theory (DFT) calculations permit to include all the different kinetic (e.g. hopping) terms at the same level. It also permits to provide a quantitative estimate of the electron-phonon coupling. Pivot in this context is the concept of deformation potential, i.e. the shift of the electronic levels upon a frozen phonon lattice distortion, which is strictly related to the magnitude of the electronphonon interaction $\frac{37}{2}$ In the context of graphenic materials, frozen phonon DFT calculations were employed to quantify in single layer systems the electron-phonon coupling associated to the modulation of the $\gamma_{0}$ term upon a lattice distortion $u \stackrel{38,39}{=}$ It can be shown indeed that the in-plane optical mode $E_{2 g}$ induces a linear splitting $\Delta \epsilon$ of the Dirac states at K point, $\Delta \epsilon \propto 6 \alpha u$, where $\alpha$ is related to the linear coupling of the electronic states with the $\mathbf{q}=0 E_{2 g}$ mode $\stackrel{36,38}{ }$ In a TB model, defining $u$ as displacement per atom, one gets $\alpha=d \gamma_{0} / d u$. DFT calculations in single-layer graphene obtain $\alpha=4.5$

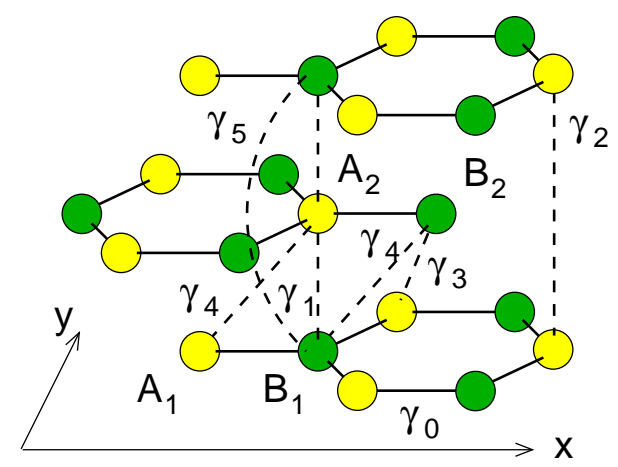

FIG. 1: (color online) (a) Atomic structure of multilayer graphene with Bernal stacking showing the relevant hopping terms $\gamma_{i}$. Atoms B1 and A2, connected by vertical $\gamma_{1}$, denoted by darker colors, contain also a local crystal field potential. 
$\mathrm{eV} / \AA .38$ A similar value $\alpha \approx 4.4 \mathrm{eV} / \AA$ is found also in graphite 38,39 where the linear energy splitting $\Delta \epsilon$ at the $\mathrm{H}$ point (where the interlayer hopping is unaffective) can be shown to be uniquely related to $\alpha=d \gamma_{0} / d u$. In both cases, in graphene and graphite, a GW theory leads to slight larger values $\alpha=5.1-5.3 \mathrm{eV} / \AA . \underline{38}$

Despite large effort has been devoted thus in literature to study the electron-phonon interaction related to the $\gamma_{0}$ term, virtually no work has been addressed so far to provide a quantitative estimate of the electron-phonon coupling associated with the modulation of the other hopping terms. A quantitative insight on this issue, on the other hand, becomes increasing important because of the role of such terms to many effects, from the establishment of unconventional anisotropic phases in strained bilayer systems $\frac{9,40}{4}$ to the evaluation of the optical properties of the in-plane and out-of-plane phonon mode in multilayer systems and in graphite. $\underline{\underline{41}}$

Aim of the present paper is to fill this gap and to provide, with a first-principle DFT calculation, a quantitative study of the electron-phonon coupling associated with the modulation of other main hopping terms, both for in-plane and for the out-of-plane vibrations. We address this issue focusing on the optical phonon modes at $\mathbf{q}=0$ in bilayer graphene. The modulation of each hopping term with the relative distance, however, permits to provide a generalization of the present results at any finite $\mathbf{q}$.

\section{FROZEN PHONON ANALYSIS}

In this paper we consider single-layer and bilayer graphene with typical Bernal stacking. We take the inplane nearest-neighbor carbon-carbon distance $b=1.42$ $\AA(a=2.46 \AA$ the lattice constant), and the interlayer distance $d=3.35 \AA$. Such lengths rule thus the magnitude of the in-plane hopping term $\gamma_{0}$ and out-ofplane hopping terms $\gamma_{i}$ on the relative distance of the corresponding atoms. For sake of simplicity, we denote with $b_{i}$ the distance associated with each hopping term in the undistorted structure, namely $b_{0}=\left|\mathbf{R}_{\mathrm{A} 1}-\mathbf{R}_{\mathrm{B} 1}\right|$, $b_{1}=\left|\mathbf{R}_{\mathrm{B} 1}-\mathbf{R}_{\mathrm{A} 2}\right|, b_{3}=\left|\mathbf{R}_{\mathrm{A} 1}-\mathbf{R}_{\mathrm{B} 2}\right|, b_{4}=\left|\mathbf{R}_{\mathrm{A} 1}-\mathbf{R}_{\mathrm{A} 2}\right|$. We assume that on the local scale the hopping terms $\gamma_{i}$ depend uniquely on the relative distance $r, \gamma_{i}=\gamma_{i}(r)$. The modulation of such hopping terms induced by the lattice displacement determines thus the electron-phonon interaction. In full generality, we define thus a electronphonon coupling as $\alpha_{i}=-d\left|\gamma_{i}\right| /\left.d r\right|_{r=b_{i}}$ Note that, since the amplitude of the hopping parameters $\left|\gamma_{i}(r)\right|$ generally decreases with increasing the distance $r$, we have introduce an explicit sign (-) in the definition of $\alpha_{i}$ so that the corresponding electron-phonon couplings is by definition chosen to be positive.

In order to reveal the electron-phonon coupling $\alpha_{i}$ for each hopping parameter $\gamma_{i}$, we consider the $E_{2 g}$ mode for the single layer graphene, and the $B_{1 g_{1}}, E_{2 g_{2}}$ and $E_{2 g_{1}}$, for the bilayer graphene as sketched in Fig. 2a.
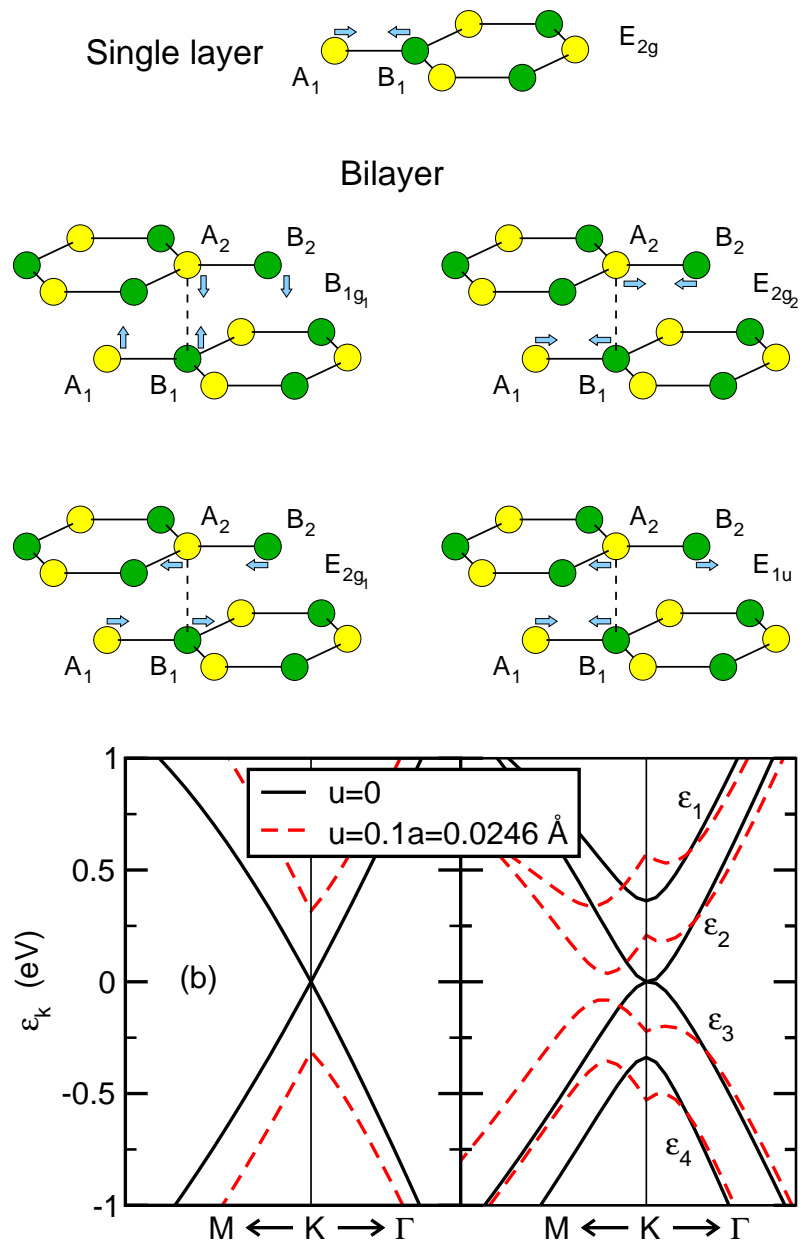

FIG. 2: (color online) (a) Sketch of atomic displacements for the relevant phonon lattice modes here considered in singlelayer graphene $\left(E_{2 g}\right)$, and in bilayer graphene $\left(B_{1 g_{1}}, E_{2 g_{2}}\right.$, $\left.E_{2 g_{1}}, E_{1 u}\right)$. (b) Representative band structure of single layer and bilayer graphene upon a frozen phonon lattice distortion (red dashed lines) with $u=0.1 a=0.0246 \AA$ for the $E_{2 g}$ mode in single layer graphene (left panel) and for the $E_{2 g_{2}}$ mode in bilayer graphene (right panel). Also shown is the undistorted band structure and the band labels $\left(\epsilon_{1}-\epsilon_{4}\right.$ from top to bottom band.

We focus on the deformation potential close to the $\mathrm{K}$ point, where one-particle low-energy excitations are involved, which makes a DFT approach particularly efficient. We compute the electronic band structure in the presence of a static frozen phonon displacement by using a plane-wave implementation ${ }^{42}$ of the density functional theory in the local-density approximation (LDA) for the exchange-correlation potential. $\underline{43}$ Ultra-soft pseudopotential for carbon was used with plane-wave (charge density) cutoff of 40 (400) Ry. A uniform wave-vector grid of $18 \times 18$ in the irreducible Brillouin-zone with coldsmearing of 0.02 Ry was sufficient to converge the calculated quantities to the required accuracy.

In order to provide a common framework for all the lattice modes of single-layer graphene and well as of bilayer 
graphene/graphite, we analyze the deformation potential as function of $u$, where $u$ represents the magnitude of the lattice displacement of each atom. We consider both degenerate in-plane modes along $x$ and $y$ directions, and the non degenerate out-of-plane modes. For each case we choose, respectively, $\mathbf{u}_{\mathrm{A} 1, x}=u_{x} \hat{\mathbf{x}}, \mathbf{u}_{\mathrm{A} 1, y}=u_{y} \hat{\mathbf{y}}$, $\mathbf{u}_{\mathrm{A} 1, z}=u_{z} \hat{\mathbf{z}}$. The displacement of the other atoms is thus univocally determined by the components of the wavevector of the phonon mode.

Representative electronic structures of the single-layer and bilayer graphene in the presence of lattice distortions are shown in Fig. 20. Focusing at the K point we can expect, according the different modes considered, an opening of a gap for the Dirac energy levels and a further modulation of the upper and lower energy bands. In the bilayer system, we label the four $\pi$-bands as $\epsilon_{1}-\epsilon_{4}$, from the top to bottom energy, as shown in Fig. 2p, and we denote $\Delta \epsilon$ the possible splitting of the Dirac state $\Delta \epsilon=\epsilon_{2}-\epsilon_{3}$ and $E=\epsilon_{1}-\epsilon_{4}$ the energy difference between the upper and lower band. We fix for convenience the energy zero of our band structure at the Dirac point of the undistorted system. It is important to stress that our procedure indeed involves only energy differences so that the absolute energy position of the band structure is irrelevant.

It is also useful to introduce here the low-energy Hamiltonian for the undistorted lattice structures. Using standard notations, the single-layer and bilayer graphenes are thus described respectively by the Hamiltonians:

$$
\begin{gathered}
\hat{H}_{\mathbf{k}}^{1 \mathrm{~L}}=\left(\begin{array}{cc}
0 & \gamma_{0} f_{\mathbf{k}} \\
\gamma_{0} f_{\mathbf{k}}^{*} & 0
\end{array}\right) \\
\hat{H}_{\mathbf{k}}^{2 \mathrm{~L}}=\left(\begin{array}{cccc}
0 & \gamma_{0} f_{\mathbf{k}} & \gamma_{4} f_{\mathbf{k}} & \gamma_{3} f_{\mathbf{k}}^{*} \\
\gamma_{0} f_{\mathbf{k}}^{*} & \delta & \gamma_{1} & \gamma_{4} f_{\mathbf{k}} \\
\gamma_{4} f_{\mathbf{k}}^{*} & \gamma_{1} & \delta & \gamma_{0} f_{\mathbf{k}} \\
\gamma_{3} f_{\mathbf{k}} & \gamma_{4} f_{\mathbf{k}}^{*} & \gamma_{0} f_{\mathbf{k}}^{*} & 0
\end{array}\right),
\end{gathered}
$$

where $f_{\mathbf{k}}=\mathrm{e}^{-i k_{x} a / \sqrt{3}}+2 \mathrm{e}^{i k_{x} a / 2 \sqrt{3}} \cos \left(k_{y} a / 2\right)$, and where $\delta$ is the difference of the crystal field probed by the B1A2 carbon atoms in the bilayer structure with respect to the A1-B2 atoms.

The band structure for the undistorted bilayer graphene is shown in Fig. 2p. Equating the TB analytical expressions with the computed DFT eigenvalues we get $\epsilon_{1}=\delta+\gamma_{1}=0.3620 \mathrm{eV}, \epsilon_{4}=\delta-\gamma_{1}=-0.3382$ $\mathrm{eV}$, which permits to evaluate the parameters $\delta=0.0119$ $\mathrm{eV}$ and $\gamma_{1}=0.3501 \mathrm{eV}$.

\section{A. Single-layer graphene}

$$
\text { 1. } E_{2 g} \text { mode }
$$

With these notations, we can now consider, as a preliminary check, the frozen phonon Hamiltonian of the single-layer graphene upon the $E_{2 g}$ distortion. Along the

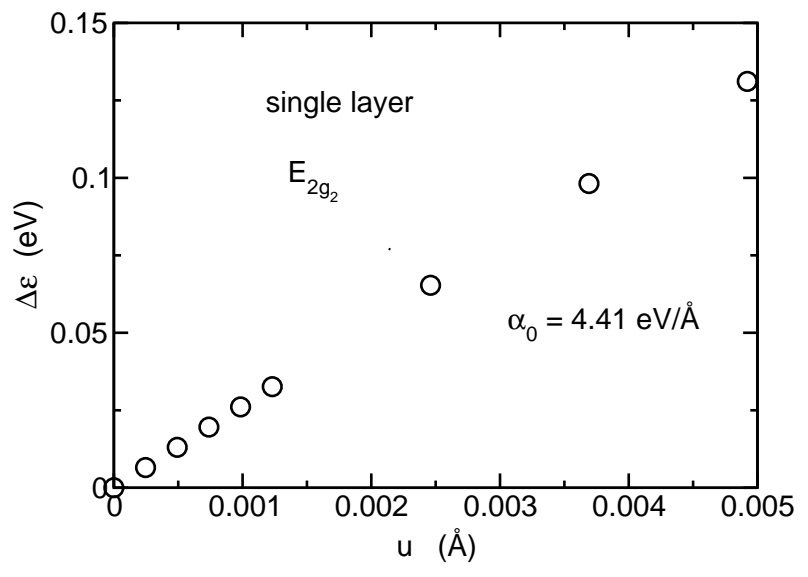

FIG. 3: Splitting of the Dirac point $\Delta \epsilon$ as a function of the $E_{2 g}$ lattice distortion $(u)$ in single layer graphene as evaluated from frozen phonon DFT calculations. The slope of $\Delta \epsilon$ vs. $u$ gives $\alpha_{0}=4.41 \mathrm{eV} / \AA$.

$x$-direction we have thus:

$$
\hat{H}_{\mathbf{k}}^{E_{2 g}}\left(u_{x}\right)=\left(\begin{array}{cc}
0 & \gamma_{0} f_{\mathbf{k}}+\alpha_{0} g_{\mathbf{k}} u_{x} \\
\gamma_{0} f_{\mathbf{k}}^{*}+\alpha_{0} g_{\mathbf{k}}^{*} u_{x} & 0
\end{array}\right),
$$

where $g_{\mathbf{k}}=2 \mathrm{e}^{-i k_{x} a / \sqrt{3}}-2 \mathrm{e}^{i k_{x} a / 2 \sqrt{3}} \cos \left(k_{y} a / 2\right)$. At the $\mathrm{K}=4 \pi / 3 a(0,1)$ point, we get $f_{\mathrm{K}}=0, g_{\mathrm{K}}=3$, so that

$$
\hat{H}_{\mathrm{K}}^{E_{2 g}}\left(u_{x}\right)=\left(\begin{array}{cc}
0 & \Delta_{0, x} \\
\Delta_{0, x} & 0
\end{array}\right),
$$

where $\Delta_{0, x}=3 \alpha_{0} u_{x}$. The degenerate levels at the Dirac point result thus splitted in single layer graphene upon a $E_{2 g}$ lattice distortion along the $x$-axis of a total amount $\Delta \epsilon^{E_{2 g}}(u)=2\left|\Delta_{0, x}\right|=6 \alpha_{0}\left|u_{x}\right|$, in agreement with Refs $29,36,38$.

A similar result can be obtained by considering lattice displacements along the $y$-direction. In this case we have:

$$
\hat{H}_{\mathbf{k}}^{E_{2 g}}\left(u_{y}\right)=\left(\begin{array}{cc}
0 & \gamma_{0} f_{\mathbf{k}}+\alpha_{0} h_{\mathbf{k}} u_{y} \\
\gamma_{0} f_{\mathbf{k}}^{*}+\alpha_{0} h_{\mathbf{k}}^{*} u_{y} & 0
\end{array}\right),
$$

where $h_{\mathbf{k}}=i 2 \sqrt{3} \mathrm{e}^{i k_{x} a / 2 \sqrt{3}} \sin \left(k_{y} a / 2\right)$. At the $\mathrm{K}=$ $4 \pi / 3 a(0,1)$ point, we get $h_{\mathrm{K}}=3 i$, so that

$$
\hat{H}_{\mathrm{K}}^{E_{2 g}}\left(u_{y}\right)=\left(\begin{array}{cc}
0 & i \Delta_{0, y} \\
-i \Delta_{0, y} & 0
\end{array}\right),
$$

with $\Delta \epsilon^{E_{2 g}}(u)=2\left|\Delta_{0, y}\right|=6 \alpha_{0}\left|u_{y}\right|$ also in this case, reflecting the perfect degeneracy of the $x$ vs $y$ in-plane lattice vibrations.

Our DFT computed splitting is shown in Fig. 3, from which we get $\alpha_{0}=4.41 \mathrm{eV} / \AA$, in nice agreement with Refs. 36, 38. We found virtually no difference for lattice distortions along $x$ or $y$ direction on this range of $u$.

\section{B. Bilayer graphene}

Once evaluated the in-plane electron-phonon coupling $\alpha_{0}$ associated with the $\gamma_{0}$ hopping term in the single- 


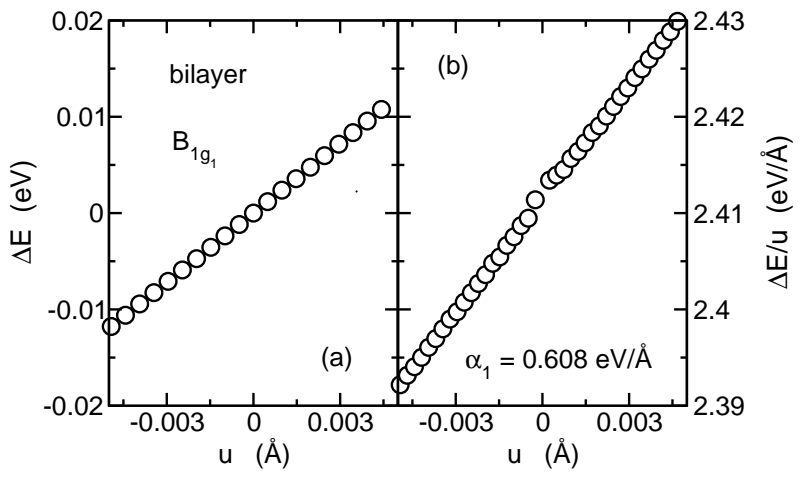

FIG. 4: (a) Energy difference $\Delta E^{B_{1 g_{1}}}(u)$ between upper and lower bands as a function of the $B_{1 g_{1}}$ lattice displacement $u_{z}$ in bilayer graphene. (b) Corresponding ratio $\Delta E^{B_{1 g_{1}}} / u$ as a function of the lattice distortion $u$. The extrapolation for $u \rightarrow 0$ gives $\alpha_{1}=0.608 \mathrm{eV} / \AA$.

layer graphene, we can now address the role of higher order hopping terms in multilayer graphenes, using the bilayer graphene as a suitable tool.

\section{1. $B_{1 g_{1}}$ mode}

We first consider the out-of-plane $B_{1 g_{1}}$ mode, as depicted in Fig. 2a. This is a quite peculiar mode since it does not lift any symmetry of the crystal. We can thus still write the four energy levels at the $\mathrm{K}$ point as

$$
\begin{aligned}
\epsilon_{2 / 3} & =0 \\
\epsilon_{1} & =\delta(u)+\gamma_{1}(u) \\
\epsilon_{4} & =\delta(u)-\gamma_{1}(u)
\end{aligned}
$$

where we have explicitly expressed the intrinsic dependence of the parameters $\delta$ and $\gamma_{1}$ on the $B_{1 g_{1}}$ lattice distortion. We can note that, as a consequence of the symmetry preserving displacements, no gap is opened at the $\mathrm{K}$ point. Useful information is however encoded in the energy difference between the high-energy bands $E^{B_{1 g_{1}}}(u)=\epsilon_{1}(u)-\epsilon_{4}(u)$ which, from Hamiltonian2, results

$$
E^{B_{1 g_{1}}}(u)=2 \gamma_{1}+4 \alpha_{1} u
$$

We can evaluate thus the electron-phonon coupling $\alpha_{1}$ from the linear dependence of $\Delta E^{B_{1 g_{1}}}(u)=\left[E^{B_{1 g_{1}}}(u)-\right.$ $E^{\left.B_{1 g_{1}}(0)\right]}$ on $u$. The calculated DFT dependence of $\Delta E^{B_{1 g_{1}}}(u)$ as a function of the vertical displacement $u_{z}$ is shown in Fig. 4 a, whereas the ratio $\Delta E^{B_{1 g_{1}}}(u) / u$ is shown in Fig. 4 $\mathrm{b}$, whose extrapolation for $u_{z} \rightarrow 0$ gives $\alpha_{1}=0.608 \mathrm{eV} / \AA$.

\section{2. $E_{2 g_{2}}$ mode}

The $B_{1 g_{1}}$ mode is quite peculiar as, since it does not lift any symmetry of the system, it does not split the Dirac energy levels at the $\mathrm{K}$ point. We have shown above however that the splitting of the additional upper and lower bands can be used to estimate the electron-phonon coupling associated with $\gamma_{1}$. Things are richer when other modes, reducing the symmetry of the crystal, are considered. In this case useful information about different electron-phonon coupling are encoded in the splitting of the Dirac point as well as in the $u$-dependence of the differences between high-energy bands, $\Delta E(u)$.

Let us consider for instance the electronic structure of the bilayer graphene under a $E_{2 g_{2}}$ lattice distortion. If we consider only the leading order linear electron-phonon couplings $\alpha_{i}$, we can thus write

$$
\hat{H}_{\mathbf{k}}^{E_{2 g_{2}}}\left(u_{x}\right)=\left(\begin{array}{cccc}
0 & \gamma_{0} f_{\mathbf{k}}+\alpha_{0} g_{\mathbf{k}} u_{x} & \gamma_{4} f_{\mathbf{k}} & \gamma_{3} f_{\mathbf{k}}^{*}-\cos \theta \alpha_{3} g_{\mathbf{k}}^{*} u_{x} \\
\gamma_{0} f_{\mathbf{k}}^{*}+\alpha_{0} g_{\mathbf{k}}^{*} u_{x} & \delta & \gamma_{1} & \gamma_{4} f_{\mathbf{k}} \\
\gamma_{4} f_{\mathbf{k}}^{*} & \gamma_{1} & \delta & \gamma_{0} f_{\mathbf{k}}+\alpha_{0} g_{\mathbf{k}} u_{x} \\
\gamma_{3} f_{\mathbf{k}}-\cos \theta \alpha_{3} g_{\mathbf{k}} u_{x} & \gamma_{4} f_{\mathbf{k}}^{*} & \gamma_{0} f_{\mathbf{k}}^{*}+\alpha_{0} g_{\mathbf{k}}^{*} u_{x} & 0
\end{array}\right)
$$

where $\cos \theta=b / \sqrt{b^{2}+d^{2}} \approx 0.39$ is a geometric factor accounting for the projection of the lattice displacement along the direction of the $\gamma_{3}$ bond. Evaluated at the $\mathrm{K}$ point, it reads

$$
\hat{H}_{\mathrm{K}}^{E_{2 g_{2}}}=\left(\begin{array}{cccc}
0 & \Delta_{0, x} & 0 & -\Delta_{3, x} \\
\Delta_{0, x} & \delta & \gamma_{1} & 0 \\
0 & \gamma_{1} & \delta & \Delta_{0, x} \\
-\Delta_{3, x} & 0 & \Delta_{0, x} & 0
\end{array}\right)
$$

The eigenvalues $\epsilon$ 's can be thus obtained from the secular equation:

$$
\begin{aligned}
& \epsilon^{2}(\delta-\epsilon)^{2}+2 \Delta_{0, x}^{2} \epsilon(\delta-\epsilon)-\epsilon^{2} \gamma_{1}^{2}+2 \Delta_{0, x}^{2} \Delta_{3, x} \gamma_{1} \\
& +\Delta_{0, x}^{4}-\Delta_{3, x}^{2}(\delta-\epsilon)^{2}+\Delta_{3, x}^{2} \gamma_{1}^{2}=0
\end{aligned}
$$

Eq. (13) predicts a linear splitting of the Dirac levels as a function of $u_{x}$. Linearizing with respect to $u_{x}$ we find:

$$
\Delta \epsilon^{E_{2 g_{2}}}=2\left|\Delta_{3, x}\right|=6 \cos \theta \alpha_{3}\left|u_{x}\right|,
$$




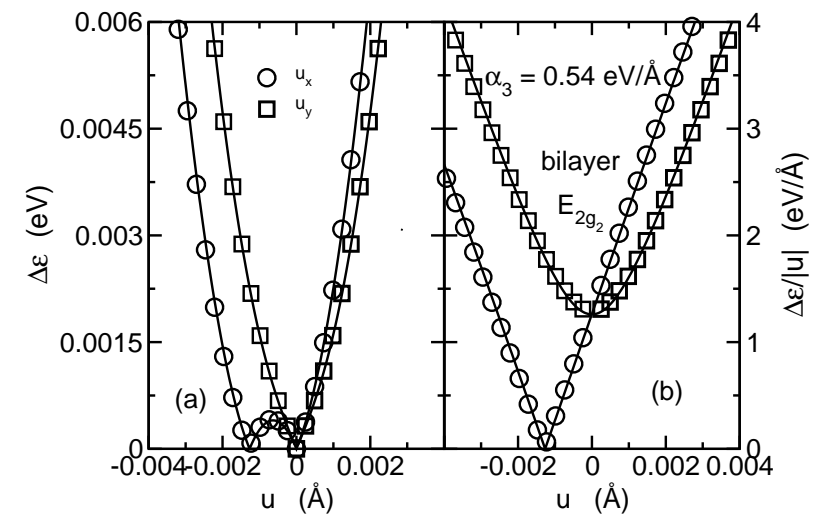

FIG. 5: (a) Splitting of the Dirac point $\Delta \epsilon$ as a function of the $E_{2 g_{2}}$ lattice distortion $u$ in bilayer graphene. Empty circles are DFT frozen phonon calculations for $u_{x}$, while empty squares for $u_{y}$. Solid lines are the solution of the corresponding analytical models in Eqs. (13), (16), using $\delta=0.0119 \mathrm{eV}$, $\gamma_{1}=0.35 \mathrm{eV}$ and $\alpha_{3}=0.54 \mathrm{eV} / \AA$. The value of $\alpha_{0} \mathrm{eV} / \AA$ is irrelevant on this quantity in this range. (b) Corresponding $\Delta \epsilon /|u|$ as a function of the lattice distortion $u$. The extrapolation of the DFT data for $u \rightarrow 0$ gives an unbiased estimate of $\alpha_{3}=0.54 \mathrm{eV} / \AA$.

which permits us to evaluate $\alpha_{3}$ from the linear splitting at the $\mathrm{K}$ point of the Dirac bands in bilayer graphene upon a $E_{2 g_{2}}$ lattice distortion. In Fig. 5 a we show the linear splitting $\Delta \epsilon_{\mathrm{K}}^{E_{2 g_{2}}}$ computed by using our frozen phonon DFT calculations for different $u_{x}$ (open circles). The linear extrapolation of $\Delta \epsilon_{\mathrm{K}}^{E_{2 g_{2}}} / u_{x}$ for $\mathrm{d} u_{x} \rightarrow 0$, as shown in Fig. $5 \mathrm{~b}$, gives us thus an unbiased estimate of $\alpha_{3}=0.54 \mathrm{eV} / \AA$.

The accuracy of such estimate, as well as of the tightbinding analysis here considered, can be checked by using this last value $\left(\alpha_{3}=0.54 \mathrm{eV} / \AA\right)$ and the TB parameters previously evaluated in an independent way in the undistorted structure $\left(\delta=0.0119 \mathrm{eV}, \gamma_{1}=0.35 \mathrm{eV}\right)$ to calculate the splitting on a wider range of $u_{x}$, without the linearization, but solving Eq. (13). The analytical results obtained in this way are in excellent agreement with DFT calculations proving thus the full intrinsic consistency of the value of $\alpha_{3}$ with respect the other TB parameters.

Note also that the DFT calculations predict a critical value $\bar{u}_{x}$ where the gap at the $\mathrm{K}$ point close, reconstructing there thus, for this particular value of $u_{x}$, a Dirac cone. This peculiar feature can also be understood using the TB model. As a matter of fact, from an inspection of Eq. (13), one can find two very close critical values $\bar{u}_{x}=-\alpha_{3} \cos \theta\left(\gamma_{1} \pm \delta\right) / 3 \alpha_{0}^{2}$ where the gap at the $\mathrm{K}$ point closes. These points are however so close that they cannot be resolved on the scale of Fig. [5 The reconstruction of the Dirac cone at the $\mathrm{K}$ point is a mixed combination of the effects of the trigonal warping induced by $\gamma_{3}$ and of the additional effects related to the lattice distortion. In the undistorted structure, indeed, we know that the effect of $\gamma_{3}$ in bilayer systems is to induce satellite Dirac cones at finite $\mathbf{k}$ in addition to the conventional one at the $\mathrm{K}$ point. Lattice distortions induce, as well as in single-layer graphene, a shift of the main Dirac point away from the $\mathrm{K}$ point, opening thus there a gap. The satellite Dirac points however move as well as functions of the lattice distortion. At a certain value, $\bar{u}_{x}$, one of the satellite Dirac points is moved again across the $\mathrm{K}$ point, and this feature is reflected in the closing of the gap in Fig. 5 at a finite $u_{x}$. The value of $\bar{u}_{x}$ agrees also in excellent way with the above analytical estimate from the tightbinding model. On the other hand, for $u_{y}$ displacements, the Dirac point moves in an orthogonal direction with respect to the $\mathrm{K}$ point and no reconstruction of Dirac cones at $\mathrm{K}$ is possible. A more detailed analysis of this issue is provided in Appendix III.

Finally, as a last check of our analysis, we computed also the frozen phonon energy splitting for $E_{2 g_{2}}$ lattice displacements along $y$. DFT calculations are shown in Fig. [5 as empty squares. To extract information about the electron-phonon coupling, we analyze the Hamiltonian at the $\mathrm{K}$ point which reads now:

$$
\hat{H}_{\mathrm{K}}^{E_{2 g_{2}}}=\left(\begin{array}{cccc}
0 & -i \Delta_{0, y} & 0 & -i \Delta_{3, y} \\
i \Delta_{0, y} & \delta & \gamma_{1} & 0 \\
0 & \gamma_{1} & \delta & -i \Delta_{0, y} \\
i \Delta_{3, y} & 0 & i \Delta_{0, y} & 0
\end{array}\right)
$$

with a secular equation:

$$
\begin{aligned}
& \epsilon^{2}(\delta-\epsilon)^{2}+2 \Delta_{0, y}^{2} \epsilon(\delta-\epsilon)-\epsilon^{2} \gamma_{1}^{2} \\
& +\Delta_{0, y}^{4}-\Delta_{3, y}^{2}(\delta-\epsilon)^{2}+\Delta_{3, y}^{2} \gamma_{1}^{2}=0 .
\end{aligned}
$$

Note that, unlike the displacements along $x$ [Eq. (13)], Eq. (16) is symmetric with respect to $u_{y} \rightarrow-u_{y}$. For small values of $u_{y}$, we once more obtain

$$
\Delta \epsilon^{E_{2 g_{2}}}=2\left|\Delta_{3, y}\right|=6 \cos \theta \alpha_{3}\left|u_{y}\right|,
$$

reflecting the degeneracy, at the linear level, of the $E_{2 g_{2}}$ mode along the two directions. The extrapolation of $\Delta \epsilon / u_{y}$ coincides with $\Delta \epsilon / u_{x}$ for $u \rightarrow 0$, providing thus the same value $\alpha_{3}=0.54 \mathrm{eV} / \AA$.

It is also interesting to give a look now at the dependence of $E^{2 g_{2}}$ at the $\mathrm{K}$ point with respect to the lattice displacement $u_{x}$. For these levels we find a quadratic dependence on $u_{x}$. Expanding Eq. (16) at the second order with respect to $u_{x}$, we get

$$
\begin{aligned}
& \epsilon_{1}=\delta+\gamma_{1}+\frac{\Delta_{0, x}^{2}}{\gamma_{1}+\delta} \\
& \epsilon_{4}=\delta-\gamma_{1}-\frac{\Delta_{0, x}^{2}}{\gamma_{1}-\delta}
\end{aligned}
$$

so that

$$
\Delta E^{2 g_{2}}=2 \gamma_{1}+\frac{2 \gamma_{1} \Delta_{0, x}^{2}}{\gamma_{1}^{2}-\delta^{2}} .
$$

The DFT calculations (open symbols) of the $u$ dependence of $\Delta E^{2 g_{2}}$ are shown in Fig. 6] (panel a), as 


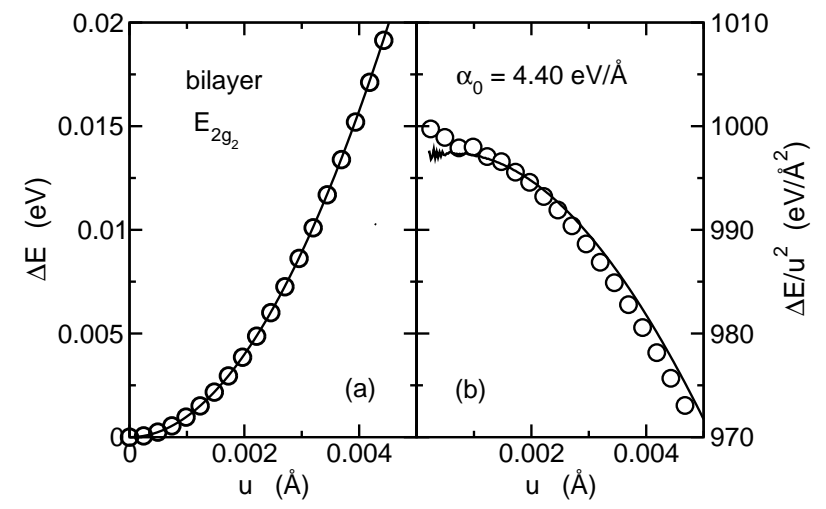

FIG. 6: (a) Variation of $\Delta E$ as a function of the $E_{2 g_{2}}$ lattice distortion $u_{x}$ in bilayer graphene. (b) Corresponding ratio $\Delta E / u^{2}$. The extrapolation for $u_{x} \rightarrow 0$ gives $\alpha_{0}=4.40 \mathrm{eV} / \AA$. Empty circles are DFT data, while solid lines are obtained from the TB model with $\delta=0.0119 \mathrm{eV}, \gamma_{1}=0.35 \mathrm{eV}$ and $\alpha_{0}=4.40 \mathrm{eV} / \AA$ and $\alpha_{1}=0.68 \mathrm{eV} / \AA$. The value of $\alpha_{3} \mathrm{eV} / \AA$ is irrelevant on this quantity in this range.

well with the ratio $\Delta E^{2 g_{2}} / u_{x}^{2}$ (panel b). The extrapolation of $\Delta E^{2 g_{2}} / u_{x}^{2}$ for $u_{x} \rightarrow 0$ provides thus an estimate $\alpha_{0}=4.40 \mathrm{eV} / \AA$ which essentially coincides with the value extracted in the single-layer graphene.

It should be here noted that Eq. (20) has been derived from Eq. (11) where only the linear terms in $u$ where retained. Some care is however needed on this regards since we are actually investigating here a quadratic dependence on $u$. A careful analysis shows that further corrections at the quadratic order in Eq. (20) appear through the explicitly dependence of $\gamma_{1}$ on $u$. Taking into account the geometry of the lattice displacement, one should write thus

$$
\Delta E^{2 g_{2}}=2 \gamma_{1}-\frac{4 \alpha_{1} u_{x}^{2}}{c}+\frac{2 \gamma_{1} \Delta_{0, x}^{2}}{\gamma_{1}^{2}-\delta^{2}}
$$

The correction coming from $\alpha_{1}$ are however two orders of magnitude smaller that the term $\propto \Delta_{0, x}$ and they are here ineffective.

\section{3. $E_{2 g_{1}}$ mode}

After having determined the electron-phonon coupling $\alpha_{0}, \alpha_{1}, \alpha_{3}$ in bilayer graphene from the frozen phonon dependence of the energy levels at the $\mathrm{K}$ point under $B_{1 g_{1}}$ and $E_{2 g_{2}}$ distortions, we are now aiming to a corresponding characterization of the last remaining parameter $\alpha_{4}$ associated with the $\gamma_{4}$ hopping. The most straightforward way to probe it, as we are going to see, is to consider the $E_{2 g_{1}}$ phonon mode, as depicted in Fig. 2

Upon distortion along the $\mathrm{E}_{2 g_{1}}$ phonon mode, the Hamiltonian reads:

$$
\hat{H}_{\mathrm{K}}^{E_{2 g_{1}}}\left(u_{x}\right)=\left(\begin{array}{cccc}
0 & \gamma_{0} f_{\mathbf{k}} & \gamma_{4} f_{\mathbf{k}}+\cos \theta \alpha_{4} g_{\mathbf{k}} u_{x} & \gamma_{3} f_{\mathbf{k}}^{*}-\cos \theta \alpha_{3} g_{\mathbf{k}}^{*} u_{x} \\
\gamma_{0} f_{\mathbf{k}}^{*} & \delta & \gamma_{1} & \gamma_{4} f_{\mathbf{k}}+\cos \theta \alpha_{4} g_{\mathbf{k}} u_{x} \\
\gamma_{4} f_{\mathbf{k}}^{*}+\cos \theta \alpha_{4} g_{\mathbf{k}}^{*} u_{x} & \gamma_{1} & \delta & \gamma_{0} f_{\mathbf{k}} \\
\cos \theta \gamma_{3} f_{\mathbf{k}}-\alpha_{3} g_{\mathbf{k}} u_{x} & \gamma_{4} f_{\mathbf{k}}^{*}+\cos \theta \alpha_{4} g_{\mathbf{k}}^{*} u_{x} & \gamma_{0} f_{\mathbf{k}}^{*} & 0
\end{array}\right)
$$

Evaluated at the K point, we thus have:

$$
\hat{H}_{\mathrm{K}}^{E_{2 g_{1}}}=\left(\begin{array}{cccc}
0 & 0 & \Delta_{4, x} & -\Delta_{3, x} \\
0 & \delta & \gamma_{1} & \Delta_{4, x} \\
\Delta_{4, x} & \gamma_{1} & \delta & 0 \\
-\Delta_{3, x} & \Delta_{4, x} & 0 & 0
\end{array}\right)
$$

where $\Delta_{4, x}=3 \cos \theta \alpha_{4} u_{x}$, and we can write the secular equation:

$$
\begin{aligned}
& \epsilon^{2}(\delta-\epsilon)^{2}+2 \cos ^{2} \theta \Delta_{4, x}^{2} \epsilon(\delta-\epsilon)-\epsilon^{2} \gamma_{1}^{2} \\
& +2 \cos ^{3} \theta \Delta_{4, x}^{2} \Delta_{3, x} \gamma_{1}+\cos ^{4} \theta \Delta_{4, x}^{4}-\cos ^{2} \theta \Delta_{3, x}^{2}(\delta-\epsilon)^{2} \\
& +\cos ^{2} \theta \Delta_{3, x}^{2} \gamma_{1}^{2}=0 .
\end{aligned}
$$

Eq. (24), predicts also, as (13), a linear splitting of the Dirac level upon lattice distortion associated once more with $\alpha_{3}$, i.e. $\Delta \epsilon_{\mathrm{K}}^{E_{2 g_{1}}}=6 \cos \theta \alpha_{3}\left|u_{x}\right|$. The value of $\alpha_{3}$ estimated upon such lattice distortion coincides with the one obtained previously using the $E_{2 g_{2}}$ mode, corroborating thus the analysis.

More useful information is however encoded in the frozen phonon dependence of $\Delta E$. Such splitting was above employed to estimate directly $\alpha_{0}$ from the frozen phonon $E_{2 g_{2}}$ lattice distortion. In the present $E_{2 g_{1}}$ context, we can see that we still get, although not a direct, an indirect estimate of $\alpha_{4}$ from the $u$-dependence of $\Delta E$. We can indeed write

$$
\epsilon_{1}=\delta+\gamma_{1}+\frac{\Delta_{4, x}^{2}}{\gamma_{1}+\delta}
$$

and

$$
\epsilon_{4}=\delta-\gamma_{1}-\frac{\Delta_{4, x}^{2}}{\gamma_{1}-\delta},
$$

so that $\Delta E$ is expected once more to presents a quadratic dependence on $u$. Taking into account, just as in the 


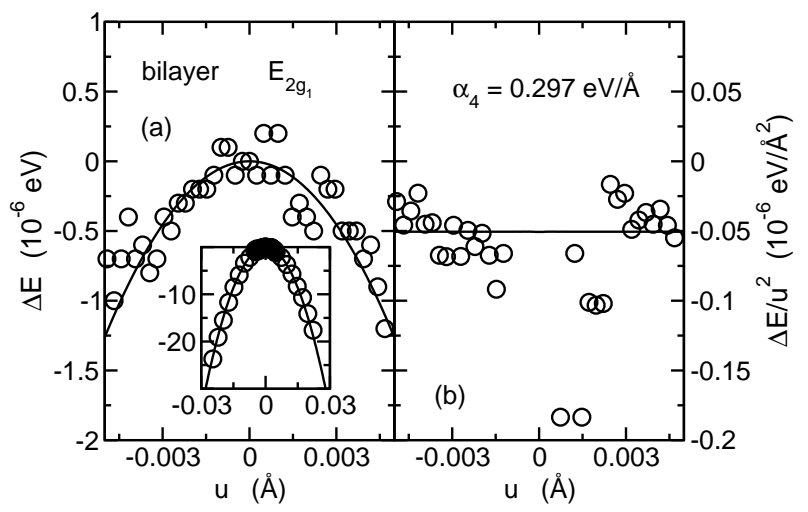

FIG. 7: (a) $\Delta E$ as a function of the $E_{2 g_{1}}$ lattice distortion $u_{x}$ in bilayer graphene. Inset: same on a wider $u$-region. Axis labels in the inset are the same as in the main panel. (b) Corresponding ratio $\Delta E / u_{x}^{2}$. From the extrapolation for $u_{x} \rightarrow 0$, and taking into account the contribution of the $u$ dependence of $\gamma_{1}$, we can estimate $\alpha_{4}=0.32 \mathrm{eV} / \AA$. Empty circles are DFT data, while solid lines are obtained from the TB model with $\delta=0.0119 \mathrm{eV}, \gamma_{1}=0.35 \mathrm{eV}$ and $\alpha_{1}=0.61$ $\mathrm{eV} / \AA$ and $\alpha_{4}=0.30 \mathrm{eV} / \AA$. The value of $\alpha_{0} \mathrm{eV} / \AA$ is irrelevant on this quantity in this range.

$E_{2 g_{2}}$ case, the quadratic dependence associated with $\gamma_{1}$, we can write thus

$$
\Delta E^{2 g_{1}}=2 \gamma_{1}-\frac{4 \alpha_{1} u_{x}^{2}}{c}+\frac{2 \gamma_{1} \Delta_{4, x}^{2}}{\gamma_{1}^{2}-\delta^{2}}
$$

DFT calculations for $\Delta E^{2 g_{1}}$ are shown in Fig. [7a on the same $u$-scale employed for other lattice modes. Due to the smallness of such $u$-dependence, numerical noise is here much larger than in previous analyses. A negative quadratic curvature can be however still clearly observed, which is better visible in a larger $u$-window in the inset. Such negative curvature is at odds with the $u$-dependence of $\Delta E^{2 g_{1}}$ coming from the contribution alone of $\alpha_{4}$ as predicted in Eq. (27). This suggests that the negative contribution from $\gamma_{1}$ is here of the same order of the term $\propto \Delta_{4, x}^{2}$. On the other hand, the $\alpha_{1}$ term alone would give an extrapolation of the ratio $\Delta E / u_{x}^{2}$ at $u_{x} \rightarrow 0$ of the order $\lim _{u_{x} \rightarrow 0} \Delta E / u_{x}^{2} \approx-0.73 \mathrm{eV} / \AA^{2}$ much bigger than what observed As a matter of fact, we can nicely reproduce the DFT data by taking $\alpha_{4}=0.30 \mathrm{eV} / \AA$. The comparison between DFT calculations and the TB model with this value of $\alpha_{4}$ reasonably good, as shown in Fig. 7. We have to stress however that, unlikely the other parameters $\alpha_{i}$ that were obtained in a direct unbiased way by a high-precision extrapolation for $u \rightarrow 0$, since $\alpha_{4}$ was deducted in an indirect way from the knowledge of $\alpha_{1}$, and given the numerically scattered DFT data in Fig. [7, this value $\alpha_{4}=0.30 \mathrm{eV} / \AA$ must be considered just as an indicative electron-phonon coupling for this hopping parameter.

\section{Other modes $\left(E_{1 u}, B_{1 g_{2}}, \ldots\right)$}

Other optical modes at $\mathbf{q}=0$ can be in principle considered to investigate the deformation potential due the electron-phonon interaction. However, they result to be not particularly convenient in order to disentangle the role of the different electron-phonon couplings associated with the different hopping parameters. Once can see for instance that the $E_{1 u}$ (also shown in Fig. 2) induces a quadratic splitting of the Dirac point as a function of $u$, whose curvature depends on the same level on both $\alpha_{0}$ and $\alpha_{4}$, so that their values cannot be estimated in an unbiased way from an extrapolation for $u \rightarrow 0$. Similar problems appear when considering the splitting of high energy bands for $E_{1 u}$, or the energy splitting (Dirac point as well as high-energy bands) for the other modes. Also in these cases, the deformation potential results to be a mixing of different electron-phonon coupling, making the quantitative evaluation of the $\alpha_{i}$ from these modes not reliable. We have however checked, on the other hand, that the above values estimated from the $B_{1 g_{1}} E_{2 g_{2}}$ and $E_{2 g_{1}}$ modes reproduce the energy differences of the electronic bands at the $\mathrm{K}$ point upon other different lattice modes.

\section{DISCUSSION AND CONCLUSIONS}

In this paper we have employed a combined TB and DFT approach to evaluate the deformation potential in single-layer and bilayer graphene associated with the modulation of the different hopping parameters. In order to avoid any fitting procedure, we have focused on the low-energy levels $\epsilon_{\nu}$ at the high-symmetry point $\mathrm{K}$ and we have characterized the electron-phonon coupling $\alpha_{i}$ for each hopping term by a careful analysis of the frozenphonon dependence of $\epsilon_{\nu}$ upon the lattice displacement for different lattice modes. In this way we were able to determine within a unique framework all the deformation potentials $\alpha_{i}$ for both the intralayer $(i=0)$ and interlayer hoppings $(i=1,3,4)$ as well as the TB parameters $\gamma_{1}, \delta$. We summarize in Table 1 our results for $\alpha_{i}$. We can also compare these values with the estimates of the absolute magnitude of the corresponding hopping parameters, as reported in the right column in Table I] The correlation between these two quantities is also shown in Fig. 8 which reveals an almost perfect linear scaling of $\alpha_{i}$ with $\gamma_{i}$. A mean-square fitting procedure gives

$$
\alpha_{i}=A+B \gamma_{i}
$$

where $A=0.141 \mathrm{eV} / \AA$ and $B=1.365 \AA^{-1}$. We would like to stress the importance of such robust underlying correlation between the magnitude of the hopping term and the corresponding electron-phonon interaction independently on the precise value of $\gamma_{i}$. It is indeed well known that the estimates of the hopping parameters $\gamma_{i}$ can significantly depend on the fitting procedure as well 


\begin{tabular}{ccc}
\hline \hline$i$ & $\alpha_{i}(\mathrm{eV} / \AA)$ & $\left|\gamma_{i}\right|(\mathrm{eV})$ \\
\hline $0(1 \mathrm{~L})$ & 4.41 & $3.12^{*}$ \\
$0(2 \mathrm{~L})$ & 4.40 & $3.12^{*}$ \\
$1(2 \mathrm{~L})$ & 0.61 & $0.35^{\dagger}$ \\
$3(2 \mathrm{~L})$ & 0.54 & $0.29^{*}$ \\
$4(2 \mathrm{~L})$ & 0.30 & $0.12^{*}$ \\
\hline
\end{tabular}

* From Ref. ${ }^{44}$

$\dagger$ present work

TABLE I: Electron-phonon coupling $\alpha_{i}$ associated with each hopping parameter $\gamma_{i}$ in single layer $(1 \mathrm{~L})$ and bilayer $(2 \mathrm{~L})$ graphene. We also show, in the right column, representative values of the TB parameters $\gamma_{1}$. We provide an estimate of $\gamma_{1}$, while $\gamma_{i}$ for $i=0,3,4$ are taken from Ref. 44 .

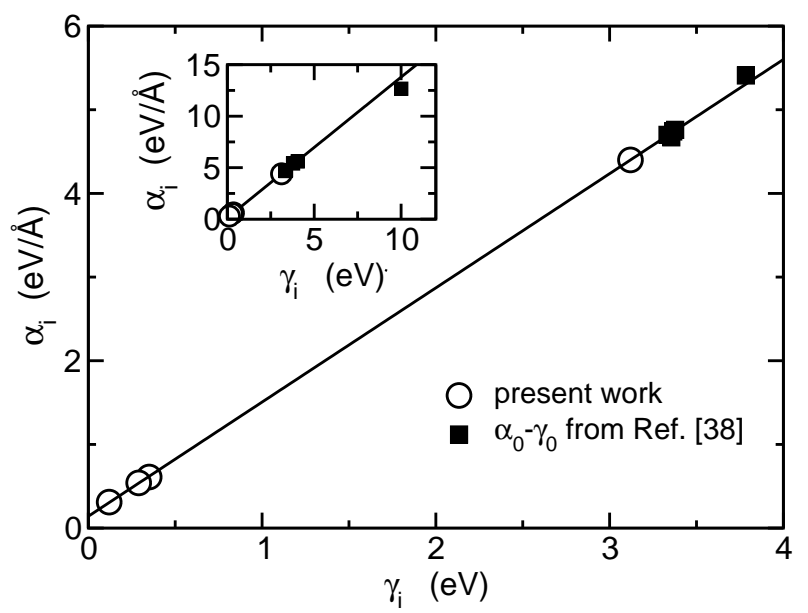

FIG. 8: Plot of the $\alpha_{i}$ vs. $\gamma_{i}$ parameters obtained from different approaches. Empty circles are data obtained by the present work where $\alpha_{i}$ was estimated by the frozen-phonon technique and $\gamma_{i}$, when not available, were taken from Ref. 44 . Filled squares are data collected by Ref. 38 using a wide variety of techniques, including Hartree-Fock, the hybrid B2LYP functional, LDA, GGA and GW. Inset: same data on a larger scale.

as on the inclusion of many-body effects in first-principles band structure for example, within the GW scheme. A detailed study of this issue, including also Hartree-Fock (HF) calculations, is provided in Ref. 38,45, where they also estimate within the same level of approximation the overall electronic $\pi$-bandwidth, related to $\gamma_{0}$, and the electron-phonon coupling $\alpha_{0}$ in single-layer graphene and graphite. Their results are also plotted in Fig. 8, where we have translated the high-energy $\pi$-band splitting $\Delta \epsilon_{\mathrm{M}}$ at the $\mathrm{M}$ point in the hopping parameter through the phenomenological relation $\Delta \epsilon_{\mathrm{M}}=1.21 \gamma_{0}$. Also in this case, considering the widest variety of approaches (HF, LDA, GGA, hybrid B3LYP functional and GW), the trend is almost perfectly linear.

Apart the fundamental implications of this result, it suggests a well, defined way to estimate experimen- tally the size of the electron-phonon coupling once the band parameters $\gamma_{i}$ are extracted experimentally, for instance by means angle-resolved photoemission spectroscopy (ARPES). In particular, the evolution of the electron-phonon coupling can be followed as a function of doping, applied electric-field, strain, etc... This can be can in a quite easy and safe way for $\gamma_{0}$, by looking at the linear conical dispersion at the $\mathrm{K}$ point, and for $\gamma_{1}$, by looking at the upper and lower band energy splitting at the same $\mathrm{K}$ point in bilayer graphene and graphite. Experimental determinations of $\gamma_{3}$ and $\gamma_{4}$ have been also provided in literature.

Our analysis provides thus a crucial, and previously missing, information to include quantitatively the role of the lattice deformations on the electronic, transport and optical properties of multilayered graphene. The effects of the lattice deformations on the electronic structure can be included in TB models involving the deformation potential associated with higher hopping terms than the nearest-neighbor ones.

\section{Acknowledgments}

E.C. acknowledges support from the European FP7 Marie Curie project PIEF-GA-2009-251904 and G.P. from CINECA-HPC ISCRA supercomputing grant.

\section{Appendix A: Dirac cone reconstruction upon $E_{2 g_{s}}$ lattice distortion}

In this Appendix we discuss in more details the origin and the phenomenology of the reconstruction of the Dirac point at the $\mathrm{K}$ edge for a critical value of the $E_{2 g_{2}}$ lattice distortion, as pointed out by DFT calculations in Fig. 6 and confirmed by the TB model.

As a starting point we remind that in realistic undistorted bilayer graphenes, electronic processes like the "skew" hopping $\gamma_{3}$ split the the parabolic Dirac cone in four linear Dirac points $\underline{46}$ In the simplest TB model with only $\gamma_{0}-\gamma_{1}-\gamma_{3}$ hoppings, the four Dirac points are located respectively at $\mathbf{k}=(0,0),\left(k_{3}, 0\right),\left(-k_{3} / 2, \sqrt{3} k_{3} / 2\right)$, $\left(-k_{3} / 2,-\sqrt{3} k_{3} / 2\right)$, where $k_{3}=\gamma_{1} \gamma_{3} / \gamma_{0} \hbar v_{\mathrm{F}}, \underline{46}$

In order to investigate the role of the $E_{2 g_{2}}$ lattice distortion, we expand the Hamiltonian (11) for small but finite $\mathbf{k}=\left(k_{x}, k_{y}\right)$. Neglecting here for simplicity the terms $\gamma_{4}, \delta$ that break the particle-hole symmetry, we can thus write:

$$
\hat{H}_{\mathbf{k}}^{E_{2 g_{2}}}\left(u_{x}\right)=\hbar v_{\mathrm{F}}\left(\begin{array}{cccc}
0 & \pi_{0, u} & 0 & v_{3} \pi_{3, u}^{*} \\
\pi_{0, u}^{*} & 0 & \tilde{\gamma}_{1} & 0 \\
0 & \tilde{\gamma}_{1} & 0 & \pi_{0, u} \\
v_{3} \pi_{3, u} & 0 & \pi_{0, u}^{*} & 0
\end{array}\right)
$$

where $\pi_{0, u}=k_{x}+i k_{y}+a_{0} u_{x}, \pi_{3, u}=k_{x}+i k_{y}-a_{3} u_{x}$, and where $a_{0}=3 \alpha_{0} / \hbar v_{\mathrm{F}}, a_{3}=3 \alpha_{3} \cos \theta / \hbar v_{\mathrm{F}}, \tilde{\gamma}_{1}=\gamma_{1} / \hbar v_{\mathrm{F}}$. 


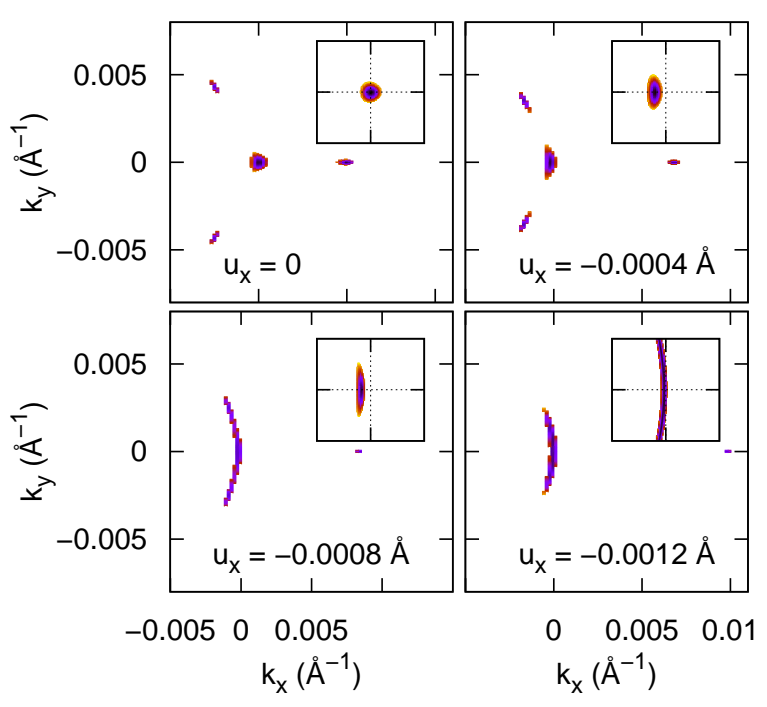

FIG. 9: (color online) Evolution of the low-energy Dirac-like states $(\epsilon \approx 0)$ as a function of the $E_{2 g_{2}}$ lattice displacements $u_{x}$. Inset: a zoom in the region $\left|k_{x}\right|,\left|k_{y}\right| \leq 0.001 \AA^{-1}$. Colors refer here to the energy distance from the Dirac points at $\epsilon=0$, The color scale has been adapted in each panel to make more visible the low energy states, with $\epsilon=0$ being the darked regions.

In the absence of particle-hole asymmetry, the four Dirac cones lie at the same energy $\epsilon=0$ also in the presence of lattice distortion. We can thus trace their evolution as a function of $u_{x}$ by analyzing the solution

$$
\operatorname{det}\left[\hat{H}_{\mathbf{k}}^{E_{2 g_{2}}}\left(u_{x}\right)\right]=0
$$

The evolution of the Dirac points, corresponding to the low-energy states of (A2), as a function of $u_{x}$, in the relevant region $u_{x}<0$, is shown in Fig. 9, The inset shows also a zoom close to the $\mathrm{K}$ point. In similar way as it has been reported for uniaxial strain,,$\frac{9}{}$ also upon the optical $E_{2 g_{2}}$ lattice distortion the Dirac points shift away from their original location for $u=0$. While such shift is monotonic for the three "leg parts", the shift of the central one is however non monotonic, with a initial departure from the K point, followed by a turn back along the opposite direction. Hence, at a critical value $\bar{u}_{x}=$ $-\tilde{\gamma}_{1} a_{3} / a_{0}^{2}$ the "central part" will eventually cross again the $\mathrm{K}$ point and then continue moving on the opposite side.

We can quantify this evolution by focusing on the axis $k_{x}$ and tracing the evolution of the roots of Eq. (A2) for $k_{y}=0$. A straightforward analysis gives thus:

$$
\begin{aligned}
\bar{k}_{x, \pm}= & \frac{\tilde{\gamma}_{1} v_{3}-2 a_{0} u_{x}}{2} \\
& \pm \frac{1}{2} \sqrt{\tilde{\gamma}_{1}^{2} v_{3}^{2}-4 \tilde{\gamma}_{1} v_{3} a_{0} u_{x}-4 \tilde{\gamma}_{1} a_{3} u_{x}}
\end{aligned}
$$

where $\bar{k}_{x,-}$ is the non monotonic solution for $u_{x}<0$ starting from $\mathbf{k}=(0,0)$ at $u_{x}=0$ and $\bar{k}_{x,+}$ is the second shifting away solution starting from $\mathbf{k}=\left(k_{3}, 0\right)$. From Eq. (A3) we thus get a critical value $\bar{u}_{x}=$ $-\tilde{\gamma}_{1} a_{3} / a_{0}^{2}=-\alpha_{3} \cos \theta \gamma_{1} / 3 \alpha_{0}^{2}$. Similar calculations can be generalized including the crystal field $\delta$ which breaks the particle-hole symmetry. We get in this case the result $\bar{u}_{x}=-\alpha_{3} \cos \theta\left(\gamma_{1} \pm \delta\right) / 3 \alpha_{0}^{2}$, as reported in Sec. IB2.
1 J.H. Chen, C. Jang, S. Xiao, M. Ishigami, and M.S. Fuhrer, Nat. Nanotech. 3, 206 (2008).

2 S. Fratini and F. Guinea, Phys. Rev. B 77, 195415 (2008).

3 E.H. Hwang and S. Das Sarma, Phys. Rev. B 77, 115449 (2008).

4 D.K. Efetov and P. Kim, Phys. Rev. Lett. 105, 256805 (2010).

5 H. Ochoa, E.V. Castro, M.I. Katsnelson, and F. Guinea, Phys. Rev. B 83, 235416 (2011).

6 M.A.H. Vozmediano, M.I. Katsnelson, and F. Guinea, Phys. Rep. 496, 109 (2010).

7 J.M.B. Lopes dos Santos, N.M.R. Peres, and A.H. Castro Neto, Phys. Rev. Lett. 99, 256802 (2007); arXiv:1202.1088 (2012).

8 F. de Juan, A. Cortijo, M.A.H. Vozmediano, and A. Cano, Nat. Phys. 7, 811 (2011).

9 M. Mucha-Kruczynski, I.L. Aleiner, and V.I. Fal'ko, Phys. Rev. B 84, 041404 (2011).

10 A.C. Ferrari, J C. Meyer, V. Scardaci, C. Casiraghi, M. Lazzeri, F. Mauri, S. Piscanec, D. Jiang, K.S. Novoselov, S. Roth, and A.K. Geim, Phys. Rev. Lett. 97, 187401 (2006).

11 S. Pisana, M. Lazzeri, C. Casiraghi, K.S. Novoselov, A.K. Geim, A.C. Ferrari, and F. Mauri, Nat. Mat. 6, 198 (2007).
12 J. Yan, Y. Zhang, P. Kim, and A. Pinczuk, Phys. Rev. Lett. 98, 166802 (2007).

13 J. Yan, E.A. Henriksen, P. Kim, and A. Pinczuk, Phys. Rev. Lett. 101, 136804 (2008).

14 L.M. Malard, D.C. Elias, E.S. Alves, and M. A. Pimenta, Phys. Rev. Lett. 101, 257401 (2008).

15 A.B. Kuzmenko, L. Benfatto, E. Cappelluti, I. Crassee, D. van der Marel, P. Blake, K.S. Novoselov, and A.K. Geim, Phys. Rev. Lett. 103, 116804 (2009).

16 T.T. Tang, Y. Zhang, C.-H. Park, B. Geng, C. Girit, Z. Hao, M.C. Martin, A. Zettl, M.F. Crommie, S.G. Louie, Y.R. Shen, and F. Wang, Nat. Nanotech. 5, 32 (2010).

17 Z.Q. Li, C.H. Lui, E. Cappelluti, L. Benfatto, K.F. Mak, G.L. Carr, J. Shan, and T.F. Heinz, arXiv:1109.6367

18 A.H. Castro Neto, F. Guinea, N.M.R. Peres, K.S. Novoselov, and A.K. Geim, Rev. Mod. Phys. 81, 109 (2009).

19 L.M. Zhang, Z.Q. Li, D.N. Basov, M.M. Fogler, Z. Hao, and M. C. Martin, Phys. Rev. B 78, 235408 (2008).

20 M. Koshino and E. McCann, Phys. Rev. B 79, 125443 (2009).

21 A.B. Kuzmenko, I. Crassee, D. van der Marel, P. Blake, and K.S. Novoselov, Phys. Rev. B 80, 165406 (2009). 
22 Z.Q. Li, E.A. Henriksen, Z. Jiang, Z. Hao, M.C. Martin, P. Kim, H.L. Stormer, and D.N. Basov, Phys. Rev. Lett. 102, 037403 (2009).

23 A.A. Avetisyan, B. Partoens, and F.M. Peeters, Phys. Rev. B 81, 115432 (2010).

24 F. Zhang, B. Sahu, H. Min, and A.H. MacDonald, Phys. Rev. B 82, 035409 (2010).

${ }^{25}$ K. Zou, X. Hong, and J. Zhu, Phys. Rev. B 84, 085408 (2011).

26 C.H. Lui, Z.Q. Li, K.F. Mak, E. Cappelluti, and T.F. Heinz, arXiv:1105.4658v1.

27 V.M. Apalkov and T. Chakraborty, arXiv:1111.3580v1.

28 M.S. Dresselhaus and G. Dresselhaus, Adv. Phys. 51, 1 (2002).

29 T. Ando, J. Soc. Phys. Jpn. 75, 124701 (2006).

30 T. Ando, J. Soc. Phys. Jpn. 76, 104711 (2007).

31 T. Ando and M. Koshino, J. Soc. Phys. Jpn. 78, 034709 (2009).

32 P. Gava, M. Lazzeri, A.M. Saitta, and F. Mauri, Phys. Rev. B 80, 155422 (2009).

33 E. Cappelluti, L. Benfatto, and A.B. Kuzmenko, Phys. Rev. B 82, 041402 (2010).

34 We mean here with "Dirac point" any point where upper and lower bands touch each other, in a semimetal way, independently of their linear or parabolic (or higher order) nature.
35 J.L. Mañes, F. Guinea, and M.A. H. Vozmediano, Phys. Rev. B 75, 155424 (2007).

${ }^{36}$ Ge. G. Samsonidze, E.B. Barros, R. Saito, J. Jiang, G. Dresselhaus, and M. S. Dresselhaus, Phys. Rev. B 75, 155420 (2007).

37 F.S. Khan and P.B. Allen, Phys. Rev. B 29, 3341 (1984)

38 M. Lazzeri, C. Attaccalite, L. Wirtz, and F. Mauri, Phys. Rev. B 78, 081406 (2008).

39 S. Piscanec, M. Lazzeri, F. Mauri, A.C. Ferrari, and J. Robertson, Phys. Rev. Lett. 93, 185503 (2004).

40 E. Mariani, A.J. Pearce, and F. von Oppen, arXiv:1110.2769

41 M. Manzardo, E. Cappelluti, and A.B. Kuzmenko, unpublished.

42 P. Giannozzi et al., J. Phys. Condens. Matter 21, 395502 (2009).

43 Appendix C of J.P. Perdew and A. Zunger, Phys. Rev. B 23, 5048 (1981).

44 B. Partoens and F.M. Peeters, Phys. Rev. B 74, 075404 (2006).

45 A. Grüneis, C. Attaccalite, L. Wirtz, H. Shiozawa, R. Saito, T. Pichler, and A. Rubio, Phys. Rev. B 78, 205425 (2008).

46 E. McCann and V.I. Fal'ko, Phys. Rev. Lett. 96, 086805 (2006). 